Supporting Information

Control of Protein Conformation and Orientation on Graphene

Shuai Wei, ${ }^{*}$ Xingquan Zou, ${ }^{*}$ Jiayi Tian, Hao Huang, Wen Guo, and Zhan Chen, ${ }^{* *}$ Department of Chemistry,

University of Michigan, Ann Arbor, Michigan 48109, United States 


\section{Spectral Fitting}

Table S1. SFG spectral fitting parameters of mutated GB1 on graphene.

\begin{tabular}{cccc}
\hline Ppp & Peak strength (a.u.) & Peak center $\left(\mathrm{cm}^{-1}\right)$ & Peak width $\left(\mathrm{cm}^{-1}\right)$ \\
\hline & -52.4 & 1635.2 & 27.3 \\
\hline & 33.2 & 1647.1 & 22.5 \\
\hline Ssp & 43.6 & 1701.8 & 54.6 \\
\hline & -25.4 & 1635.0 & 47.5 \\
\hline
\end{tabular}

Table S2. ATR-FTIR spectral fitting parameters of mutated GB1 on graphene.

\begin{tabular}{ccccc}
\hline & Intensity (a.u.) & $\begin{array}{l}\text { Peak center } \\
\left(\mathrm{cm}^{-1}\right)\end{array}$ & $\begin{array}{l}\text { Peak width } \\
\left(\mathrm{cm}^{-1}\right)\end{array}$ & Assignment \\
\hline $\mathrm{P}$ & 0.0070 & 1627 & 10.3 & $\beta$-sheet \\
\hline & 0.0056 & 1648 & 8.9 & $\alpha$-helix \\
\hline $\mathrm{S}$ & 0.0038 & 1671 & 9.9 & turns and $\beta$-sheet \\
\hline & 0.0034 & 1627 & 9.9 & $\beta$-sheet \\
\hline & 0.0034 & 1648 & 9.0 & $\alpha$-helix \\
\hline & 0.0020 & 1670 & 8.4 & turns and $\beta$-sheet \\
\hline
\end{tabular}

\section{Simulation Methods}

Karanicolas-Brooks (KB) Go-like model is used for simulating protein dynamics on surface in this work. In this model, the protein folding is determined by the sequence-dependence of the native contact interactions and backbone torsional angle potential, as well as a nonbonded potential that captures cooperative contact formation. This model uses implicit solvent effect as coded in the contact parameters, so no explicit solvent molecules were involved in simulations.

Six initial poses of the protein were obtained by rotating the original protein around different axes by $90^{\circ}$ each time. Initial structures were placed about $1 \mathrm{~nm}$ above the graphene surface at the beginning of each simulation. Each simulation consisted of 100 million steps of production with a step size of $1 \mathrm{fs}$. 
All the simulations were performed under canonical ensemble (NVT) with temperature maintained at $298 \mathrm{~K}$ by the Nosé-Hoover-Chain integration method with three thermostats of mass $10^{-26} \mathrm{~kg} \AA^{2}$. Each simulation has a very large $(40 \mathrm{~nm} * 40 \mathrm{~nm} * 40 \mathrm{~nm})$ box with periodic boundaries to avoid self-interaction of any potentially fully unfolded structure.

Table S3. Parameters applied in the surface model (Equation 1), which describe the residue-graphene surface interactions. These parameters were generated in previous work against a large benchmark experimental data set, which has been applied in many cases for different sensor surface types.

\begin{tabular}{ccccc}
\hline$\theta_{1}$ & $\theta_{2}$ & $\theta_{3}$ & $\theta_{\mathrm{s}}$ & $\theta_{\mathrm{p}}$ \\
\hline 0.2340 & 0.4936 & 0.1333 & 0.0067 & 0.0333 \\
\hline
\end{tabular}

\section{Possible Orientations of Mutated GB1 on Graphene}

Figure S1. Heap map plot with possible orientation angles of mutated GB1 on graphene deduced from SFG and ATR-FTIR measurements with $20 \%$ error.

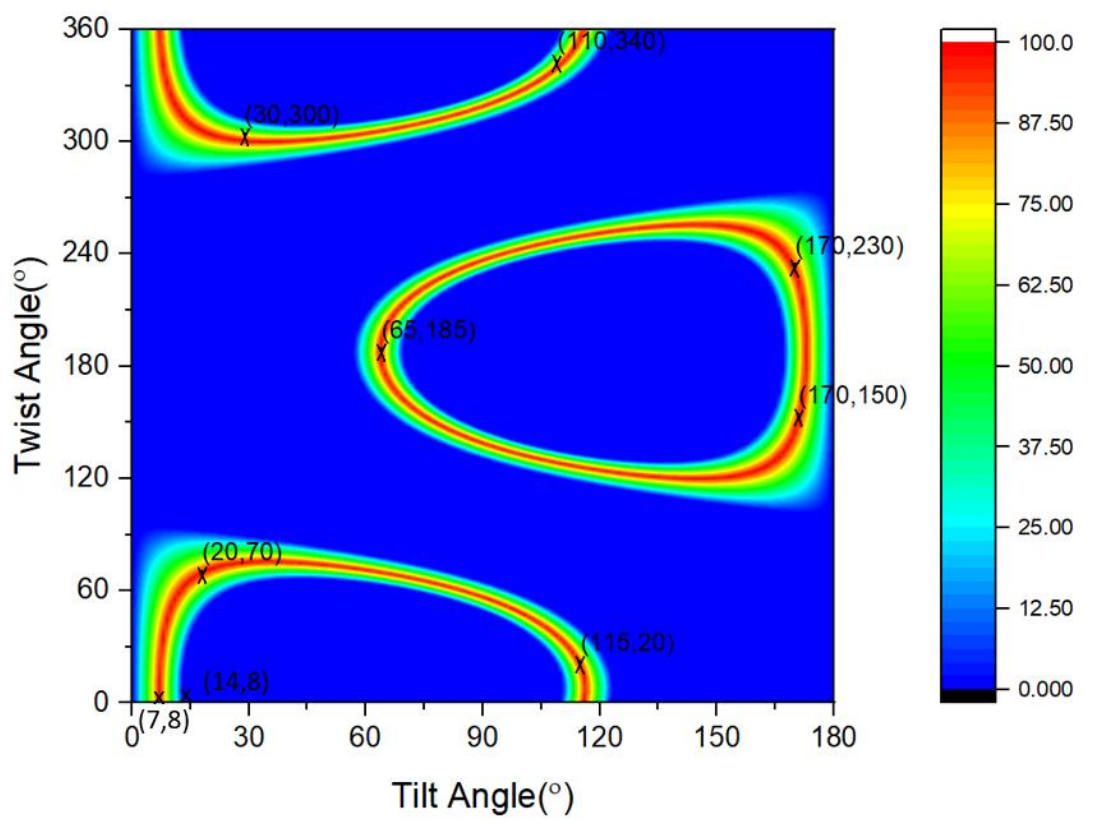


Figure S2. Possible orientations of mutated GB1 with varied tilt and twist angle combinations marked in Figure S1.

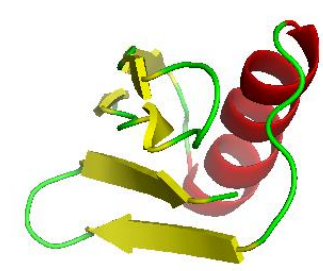

$(20,70)$

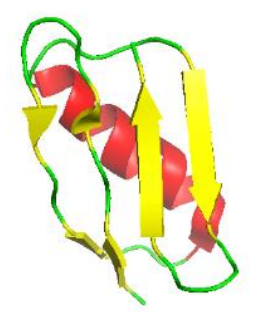

$(115,20)$

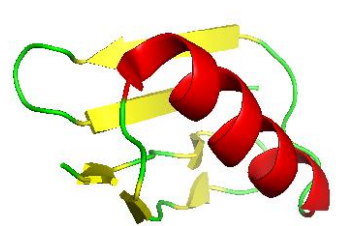

$(170,230)$

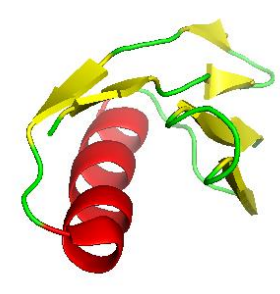

$(30,300)$

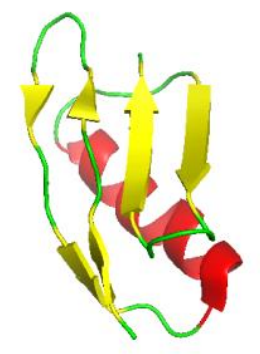

$(110,340)$

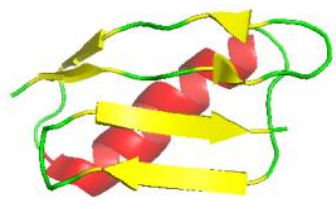

$(14,8)$

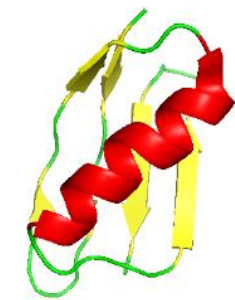

$(65,185)$
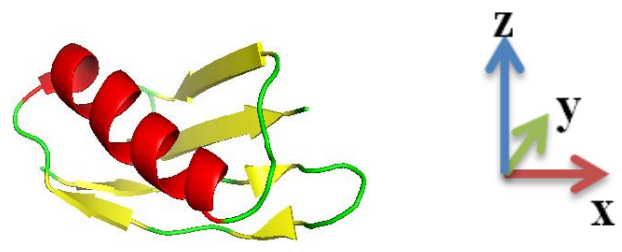

$(170,150)$

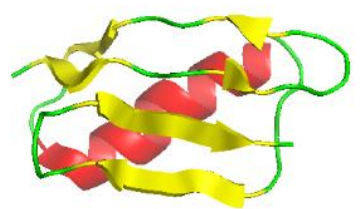

$(7,8)$

The orientation $(14,8)$ is the orientation obtained from the MD simulations. 